\title{
Dynamic Hello/Timeout timer adjustment in routing protocols for reducing overhead in MANETs
}

\author{
Nelson HERNANDEZ-CONS, Shoji KASAHARA*, Yutaka TAKAHASHI \\ Graduate School of Informatics, Kyoto University \\ 36-1, Yoshida-honmachi, Sakyo-ku, Kyoto 606-8501, Japan
}

\begin{abstract}
With the recent advancement and extensive spread of mobile devices, establishing a network with those devices has become more important than ever before. MANETs (Mobile Ad-hoc NETworks) have become a promising solution to set up a network with any mobile device. Routing in MANET is still a difficult task since the mobility of the nodes affects the local connections and makes the network topology change constantly. Traditional routing protocols in MANET work sending periodic messages to realize the changes in the topology in order to maintain the local connections up-todate. However, sending periodic messages at a fixed rate can cause overhead in situations where it can be prevented and thus reduced. In this paper, we introduce a scheme to improve the performance of the routing protocols used in MANET focusing on the reduction of the overhead. In the proposed scheme, instead of sending periodic messages at a fixed rate, we use a link change rate estimation to dynamically adjust the rate at which each node sends the control messages. The link change rate estimation is based
\end{abstract}

\footnotetext{
*Corresponding author. Tel.:+81-75-753-5493; Fax:+81-75-753-3358.

Email address: kasahara@ieee.org (Shoji KASAHARA)
} 
on the constant measurement of the local connectivity that reflects the network conditions. We evaluate the performance of our scheme using ns-2 and we compare it to other approaches to show its advantages. With the usage of our scheme, we notice a considerable reduction of the overhead without sacrificing the overall performance of the tested protocols.

Key words: MANET, soft state, overhead, AODV, OLSR, ns-2

\section{Introduction}

MANETs (Mobile Ad-hoc NETworks) are wireless mobile networks that are set up on the spot without prior preparation of any infrastructure, that is, in an ad hoc fashion. The lack of infrastructure, routers, servers and cables makes MANETs a fast, low-cost and convenient way to deploy a network with the use of mobile devices such as cellular phones, smart phones or small laptop computers. Moreover, MANETs can offer a rapid solution for a network in situations such as military operations, disaster recovery, vehicleto-vehicle communication networks, conferences, events or shows, airport terminals or for personal area networks connecting smart phones with other wearable devices.

There are several routing protocols for MANET, although very few are standardized. Routing in MANET is a difficult issue as several factors affect the stability of the network and make the topology highly dynamic. The routing protocols in MANET use several mechanisms to keep track of the changes in the network, for example, link layer sensing of the wireless links, or the usage of Hello messages at the routing level, which is referred to as soft state signaling. 
In soft state signaling, a node periodically broadcasts Hello messages to keep track of valid links and also to advertise its presence in the network. Traditional protocols use a fixed time interval to send these Hello messages, which is not optimal. For example, if the nodes in a network do not move, the links of the nodes will not change; so sending Hello messages at a fixed rate will only cause unnecessary overhead in the network. On the other hand, if the nodes are moving too fast, sending Hello messages at a fixed rate might advertise the links too late; so when a node sends a packet to a neighbor, that neighbor might not be in the same position anymore, in which case the packet will simply be dropped. The node will then have to exchange more messages to find a way to route the pending packets.

Overhead (control messages including Hello messages) is necessary to keep the nodes updated with the changes in the network. However, minimizing the overhead in MANETs is critical since the mobile nodes have a very limited amount of energy. Reducing the overhead helps the nodes to reduce the amount of energy used, so that they last longer in the network. Moreover, when the overhead is low, congestions are less likely to occur.

In this paper, we propose an overhead-reducing scheme consisting of three mechanisms to regulate the Hello timer and its timeout. First, we measure the link change rate by monitoring the number of acquired and lost links. If a node has a link change rate close to zero, its neighborhood remains unchanged. Conversely, if a node has a high link change rate, it means its neighborhood has changed. With this particular assumption, we can set the Hello timer to an adequate value. In stable networks, the value of the Hello timer can be higher so that there is no unnecessary overhead, and in highly 
dynamic networks, the value should be small to keep track of the changes in the network. With the value of the Hello timer set dynamically, we also set the Timeout timer to an adequate value. When neighbors stay an adequate amount of time in the routing tables, unnecessary route requests will not be issued, thus further reducing the overhead. Finally, when Hello messages are scheduled to be sent in the future and the network changes suddenly, we reschedule the Hello message sooner in time in order to react to the sudden changes.

We expect a considerable reduction of the overhead introduced in the network. To verify the effectiveness of the proposed scheme, we evaluate its performance using the network simulator ns-2.

The rest of this paper is organized as follows. In Section 2, we give an overview of MANET and signaling mechanisms. We also present related works on the issue of signaling and overhead in MANETs. Section 3 describes the adaptive timer scheme. Numerical examples to evaluate the effectiveness of the scheme are presented in Section 4. Finally, conclusions and future work are presented in Section 5.

\section{Related work}

In this section we present a brief overview of MANET and soft state signaling related approaches.

In soft state signaling, each node in the network sends a refresh message periodically in the form of a broadcast to all the neighboring nodes. This message is sent to refresh the state that reflects the conditions of the network. The period that a node waits until a message is sent, is called the refresh 
period. In soft state signaling, the state is automatically removed at the receivers if no refresh message is received after some specific time called the timeout period.

For the purposes of our study, we focus on the Hello timer on the sender side and on the Neighbor Timeout timer on the receiver side. The Hello timer value controls the period to wait until a Hello message needs to be sent, while the Neighbor Timeout timer controls the period of time a link towards a neighbor is considered valid. Traditional approaches use fixed values for those two timers. For example, standard AODV (7) proposes a Hello timer of one second and a timeout timer of two seconds.

Starting from the traditional approach of fixed timer values in the protocols, there have been several adaptive timer approaches for routing protocols in MANETs. Basagni et al. (1) proposed DREAM (Distance Routing Effect Algorithm for Mobility) which considers the possible usage of mobility as a way to control the refresh timer to broadcast messages. In DREAM, the authors state that a node broadcasts a control message according to its speed. The faster a node moves, the more often it broadcasts messages. Conversely, the slower a node moves, the fewer the control messages it sends. In their approach, the speed of the node, which can be obtained by mechanisms such as GPS (Global Positioning System), is the sole parameter used to control the rate to send messages. However, the individual speed of one node doesn't determine the mobility of the network. In fact, it is very difficult for a single node to decide, using only its own speed, whether the network is changing fast or the network remains stable. Moreover, the authors simulated some scenarios with all the nodes having the same speed throughout the simula- 
tions.

Camp et al. (2) proposed an approach that takes into account the transmission range of the node as well as its speed to regulate the sending rate of control messages. Moreover, there is a distinction between close nodes and far-away nodes. Close nodes receive more periodic refresh messages while far-away nodes do not receive those messages very often under the assumption that far away nodes "appear" to move more slowly from the sender's point of view. This particular model uses several constant values to modify the refresh timer.

Fast OLSR (10) by Benzaid et al. is an extension of standard OLSR that works in two modes: fast moving mode and the default normal mode. In normal mode, the protocol behaves like standard OLSR. In the fast moving mode, the protocol uses a Hello timer with a fixed smaller value obtained from simulation experiments. In (10), some scenarios are defined according to the speed of the nodes and then the values of the timers are calculated and fixed, which then makes the protocol extension not fully adaptive.

Huang et al. (4) give an algorithm based on the link change rate. A link is a local connection with a neighbor, and the link change rate measures the change in the set of links of a node through time. The link change rate is calculated using a formula given by an analytical model developed by Samar and Wicker (3). The used formula takes into account the speed of one node while the other nodes' speeds are assumed random. However, there are several parameters that have to be known for the formula to be used in real situations, such as the density of the network, the maximum and minimum speeds of the nodes and the direction of motion of the nodes. Some parameters can 
be obtained through mechanisms such as GPS (Global Positioning System) while others are more difficult to accurately estimate. Huang's algorithm is based on a Multiplicative Increase Additive Decrease (MIAD) controller to adapt the refresh timer of a protocol. His approach provides an adaptive solution to dynamically modify the refresh timer, but the dependency on network measurements to compute the parameters used in the formula for the link change rate makes it questionable for real implementations.

In (6), Sharma et al. proposed a scheme to control the timer at the sender and also at the receiver. Their approach is adaptive to the volume of control traffic and the available bandwidth on the link. The receiver has to estimate the Timeout timer from the rate at which the sender sends data, which can work in fixed wired networks but is not a reliable approach for MANET due to the dynamics of the network. In fact, in their study they focus on fixed wired networks and not wireless networks, so the methodology of their study is not directly applicable to MANETs.

\section{Proposed scheme}

In this section, we present the details of the proposed scheme to reduce the soft state overhead in MANET routing protocols. We base our study on the constant measurement of the link change rate. In the following, we consider a link as a wireless direct connection between two nodes that are neighbors and are therefore within the transmission range of each other. We also consider a stable network as a network with few topology changes, that is, the connections remain stable among the nodes. In other words, there are very few new links or lost links in the network. In general, a low mobility 
network can be considered as a stable network.

The goal of the scheme is to dynamically adjust the Hello timer and the Timeout timer according to the conditions of the network. For example, in a high mobility network (with frequent topology changes) it is desirable to use small values for the timers to quickly detect the changes in the network. On the other hand, in a low mobility network where the topology remains stable and with few changes, a large value for the timers is more effective to reduce the overhead.

In order to decide whether the mobility of the network is high or low, we use a simple way to approximate in real time the link change rate. The link change rate expresses how the number of available links changes through time and is expressed in [links/sec]. The changes occur when a new link is established or an active link is lost.

In the following, we first explain the measurement of the link change rate and then the three mechanisms in detail.

\subsection{Computation of the Link Change Rate (LCR)}

We assume each node has a neighbor table to store the addresses of its neighbors. Consider node A and node B in a MANET, both have just moved within the transmission range of each other so that they can become neighbors. Node A receives a Hello message from node B, so node A considers node $\mathrm{B}$ as a new neighbor and stores node B's address in its neighbor table. Therefore, node A has created a new link towards node B. We use a counter, newLinkCounter, to keep track of the number of new links. Similarly, if node A and node B are already neighbors, and node B departs from the MANET, node A will erase node B's entry from its neighbor table according to the 
Timeout timer. When the entry for node B is erased, node A loses the link towards node B. We use another counter, lostLinkCounter, to keep track of the number of lost links. We then calculate the link change rate $l c r$ with the following expression:

$$
l c r=\frac{\text { newLinkCounter }+ \text { lostLinkCounter }}{t},
$$

where $t$ is the time elapsed since the last measurement. It is clear that this is a very simple way to approach the link change rate. In previous approaches, in order to determine whether a node is in a high mobility or low mobility network, usually only its own speed is taken into account while the speed of the rest of the nodes is ignored or assumed random. Consider node $\mathrm{A}$ in a network and the set $N$ as the set of node A's neighbors. Let $v_{\mathrm{A}}$ and $V_{N}$ denote the speeds of node $\mathrm{A}$ and the average speed of the neighbors of $\mathrm{A}$, respectively. Notice that this approach includes both influences, $v_{\mathrm{A}}$ and $V_{N}$, since node A gains or loses links due to its own mobility, but also due to the mobility of the rest of the nodes $N$.

Instead of using an instant value of the $l c r$, we calculate an average over time using an EWMA (Exponential Weighted Moving Average) filter:

$$
L C R_{\mathrm{est}}(k)=L C R_{\mathrm{est}}(k-1) \alpha_{\mathrm{EWMA}}+L C R_{\mathrm{sample}}(k)\left(1-\alpha_{\mathrm{EWMA}}\right),
$$

where $L C R_{\text {est }}(k)$ is the average over time, $L C R_{\text {est }}(k-1)$ is the previous estimated average, $L C R_{\text {sample }}(k)$ is the most recent measured value of the link change rate calculated from (1) and $\alpha_{\text {EWMA }}$ is the parameter of the filter.

In most of the literature, the parameter $\alpha_{\mathrm{EWMA}}$ is normally adjusted to a 
fixed value. However, we follow the adaptive approach proposed by Itaya and Kasahara in (5) as it is shown in Figure 1. Since the network is constantly changing, using a constant $\alpha_{\text {EWMA }}$ may not be appropriate to deliver the best estimation values. Notice that a small value of $\alpha_{\text {EWMA }}$ provides an estimate that better reflects the current network status. With a small $\alpha_{\text {EWMA }}$, the estimated value captures rapid changes of the network, and thus it is more suited for networks changing rapidly. In other words, small $\alpha_{\text {EWMA }}$ reflects current changes while large $\alpha_{\text {EWMA }}$ reflects past estimations.

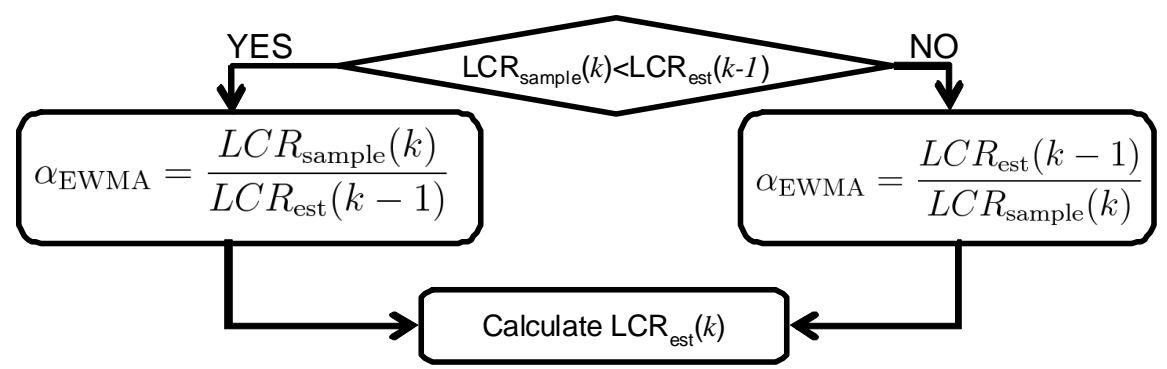

Figure 1: Adaptive calculation of $\alpha_{\text {EWMA }}$.

\subsection{Hello timer adaptive algorithm}

In order to control the Hello message sending rate we use an algorithm based on Huang's approach as the MIAD controller showed good performance in (4). However, the MIAD controller reacts slowly to high mobility networks, so we propose an algorithm based on a more aggressive controller MIMD (Multiplicative Increase Multiplicative Decrease) in order to react faster.

A flow chart of our algorithm is shown in Figure 2. We first check the lcr value. If there is a change, $l c r>0$, then we increase the rate at which the Hello messages are sent. We then calculate the value of the Hello timer and we 
start again. If there are no changes, $l c r=0$, then we proceed to decrease the refresh rate so that we send fewer Hello messages. Note that when a change is detected, the parameter $\beta$ is set to $\beta_{\max }$, so the next time we detect that there are no changes in the network, the decrease is aggressive. If the network remains stable and there are no changes, we keep decreasing the refresh rate with subsequent smaller values of $\beta$. That is, the first decrease is aggressive and the subsequent decreases are smaller because $\beta$ is also decremented by $\varepsilon$.

Note that we also take into account the value of the counter dropCount. This counter monitors the packets that are dropped due to a link failure. If an invalid link is causing packet loss, the soft state timers are not adequate to correctly match the state with the real network condition. Suppose node A and node $\mathrm{B}$ in a network. Node B then leaves the network while node A still forwards packets to it. Node A detects the link failure through the change in the $l c r$ value. Between the detection of the link failure and the discovery of an alternative link to forward the packets, the $l c r$ value might not change, so we decrease the soft state timers using the dropCount counter to detect links faster.

Finally, note from Figure 2 that the Hello timer adaptive algorithm runs in a loop. The $l c r$ measurement and this algorithm are constantly being executed.

\subsection{Adaptive Neighbor Timeout timer}

Once the Hello timer is set up according to the network mobility, we also adjust the Timeout timer that is associated to a neighbor. Recall that the Timeout timer controls the amount of time that a link towards a neighbor is 


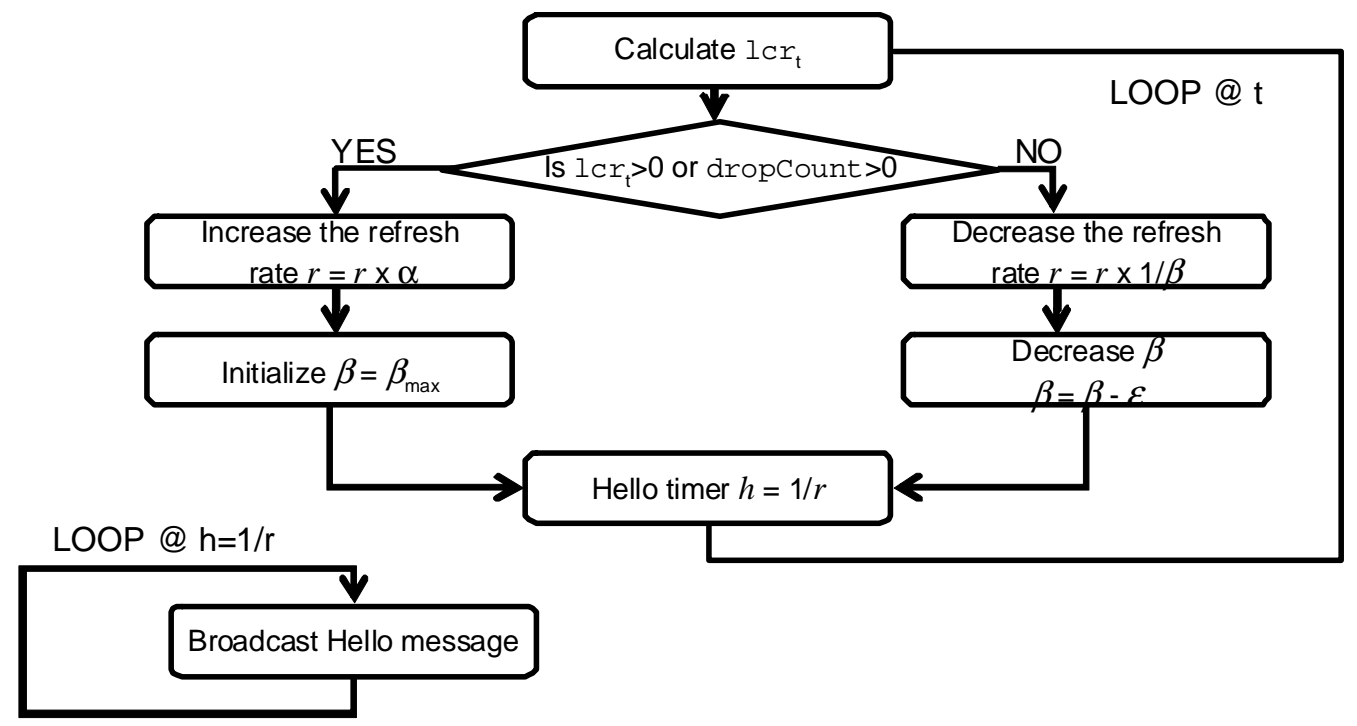

Figure 2: Algorithm to calculate the Hello timer based on the link change rate and the packet drop count.

considered valid.

The aim of this mechanism is to reduce the overhead by preventing a node from issuing a route request or search for a link. Again, consider node A and node B within the transmission range of each other. Node B sends Hello messages to node A, and node A stores node B in its neighbor table. In a stable network, if the Timeout timer for node B is small, node A will delete the entry for node $\mathrm{B}$ after a short period of time. If node $\mathrm{B}$ delays in sending Hello messages, node A will have to send a route request or search for a link which will lead to some exchange of messages from both sides. If the Timeout timer for node $\mathrm{B}$ has a higher value, node $\mathrm{A}$ can continue forwarding packets to node $\mathrm{B}$ without neither of them having to exchange control messages. Thus, in stable networks it is desirable to have a higher value of the Timeout to reduce overhead, while in highly dynamic networks 
a shorter Timeout timer value is preferable.

AODV uses the following expression to calculate the Timeout timer for each neighbor:

$$
\text { Neighbor_expire }=\text { ALLOWED_HELLO_LOSS } \times \text { HELLO_INTERVAL, }
$$

where HELLO_INTERVAL is the value of the Hello timer and its default value is set to 1000 milliseconds. The other value ALLOWED_HELLO_LOSS is a constant fixed to two. These values are fixed according to the RFC (7). Instead of using the default value of HELLO_INTERVAL, we use the dynamic adaptive value of the Hello timer calculated with our adaptive algorithm.

For OLSR the neighbor timeout timer is also set, by default, to a constant time:

$$
\text { Neighbor_Hold_Time }=3 \times \text { REFRESH_INTERVAL, }
$$

where REFRESH_INTERVAL is set to two seconds. These values are also fixed according to the RFC (9). Similar to AODV, we use the adaptive value of the Hello timer instead of the fixed REFRESH_INTERVAL value.

\subsection{Reactive Hello message scheduling}

The aim of this mechanism is to help the protocol to better react to sudden changes in the network. Recall from previous sections that the $l c r$ value is constantly being monitored and therefore, Hello messages are being scheduled to be sent in the future.

Suppose again a stable network where the Hello timer of node A has reached a high value. Consequently, the next Hello message is scheduled far in the future. If there is a sudden change in the network, it is desirable that node A should not wait until the next Hello message to react. Instead, upon 
noticing the sudden change in the network, we recalculate the Hello timer and if the value of the new Hello timer is smaller than the previous one, we reschedule the next Hello message sooner in time.

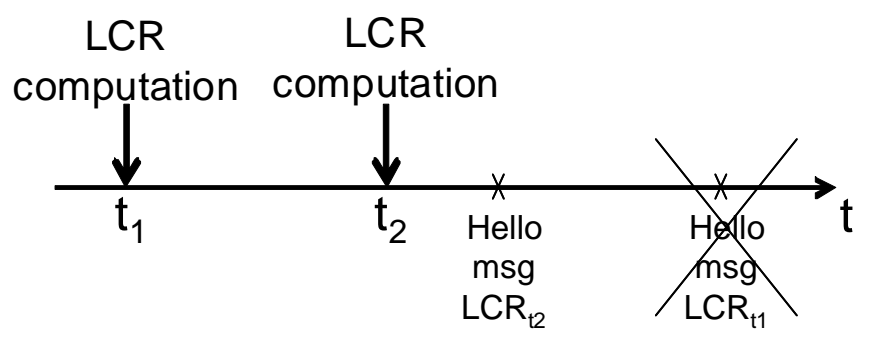

Figure 3: A Hello message scheduled at $t_{1}$ is canceled and rescheduled at $t_{2}$ as a result of sudden changes in the links.

Consider a computation of the $l c r$ at time $t_{1}$. This yields a value for the Hello timer $h_{t 1}$, and a Hello message is then scheduled at time $t_{1}+h_{t 1}$. Now, suppose at time $t_{2}$ there is a new computation of the $l c r$, and so a new value for the Hello timer $h_{t 2}$ is also calculated. If $t_{2}+h_{t 2}<t_{1}+h_{t 1}$, then the first Hello message is canceled, and a new Hello message scheduled to be sent at $t_{2}+h_{t 2}$

\section{Performance evaluation}

In this section, we evaluate the overhead reduction of the proposed scheme using the network simulator ns-2 (11) version 2.33. We simulate using two protocols, the reactive protocol AODV (7) and the proactive protocol OLSR (9). We use three different versions of these protocols: the original protocol, a modified version implementing Huang's DT_MIAD approach (4) and finally, a modified version of the protocol implementing our proposed scheme. 
For our simulations, we first generate a set of different and random scenarios. We want to cover as many situations as possible so we have nodes moving with random speeds uniformly distributed in the range $[0,40] \mathrm{m} / \mathrm{s}$. In order to measure the link change rate as a function of the speed of the nodes, we also introduce one node, namely node $\mathrm{A}$, with deterministic speed also in the range $[0,40] \mathrm{m} / \mathrm{s}$.

For each configuration of speed, we generate 100 different scenarios, and we run the three versions of each protocol with each scenario. For one scenario we run first the original protocol and we measure the link change rate, the behavior of the Hello timer and some performance metrics discussed in the next section. Then we run the exactly same scenario with the protocol implementing Huang's approach and finally with the proposed approach.

We use two different populations, $n=10$ and $n=30$ nodes to compare the density of the network. Our simulations run for 100s using CBR (Constant Bit Rate) sources sending packets of 512 bytes each, at a fixed rate of 2 packets per second. We show the detailed configuration of ns-2 in Table 1.

\subsection{Performance metrics}

In order to measure and compare the schemes, we use three performance metrics: overhead, the CBR drop rate and the end-to-end (E2E) delay.

For the overhead, we are interested in the number of packets that were received in the network, as these packets can cause collisions or congestion in the network.

The CBR drop rate represents the efficiency of the protocol to deliver the data from the source to the destination. We count the number of CBR dropped packets $\# C B R_{\text {drop }}$ and the number of CBR sent packets $\# C B R_{\text {sent }}$. 
Table 1: Basic simulation parameters.

\begin{tabular}{l|l}
\hline Parameter & Value \\
\hline Antenna type & Omni directional \\
Radio propagation & Two-ray ground \\
Mobility model & Random waypoint \\
MAC protocol & IEEE 802.11 \\
Pause time & $1[\mathrm{~s}]$ \\
Transmission range & $250[\mathrm{~m}]$ \\
Simulation area & $500 \times 500\left[\mathrm{~m}^{2}\right]$ \\
Packet size & $512[\mathrm{byte}]$ \\
Traffic model & CBR @ 8 $[\mathrm{Kb} / \mathrm{s}]$ \\
Simulation time & $100[\mathrm{~s}]$ \\
Routing protocols & AODV, OLSR \\
\hline
\end{tabular}

We calculate the $C B R_{\text {droprate }}$ with the expression

$$
C B R_{\text {droprate }}=\frac{\# C B R_{\text {drop }}}{\# C B R_{\text {sent }}} \text {. }
$$

The third measure is the end-to-end (E2E) delay. For the CBR packets, we measure the time one packet takes to reach its final destination. We use the following expression to calculate the E2E delay:

$$
E 2 E_{\text {delay }}=t_{\text {recv }}(p k t I D)-t_{\text {sent }}(p k t I D)
$$

where $\operatorname{pktID}$ is a number that uniquely identifies a packet, and $t_{\mathrm{recv}}$ and $t_{\text {sent }}$ are the times where the packet was received and sent, respectively. 


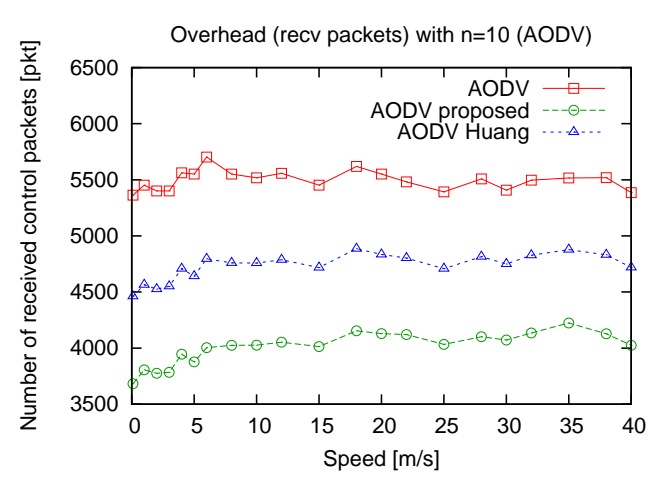

(a) Low density $n=10$.

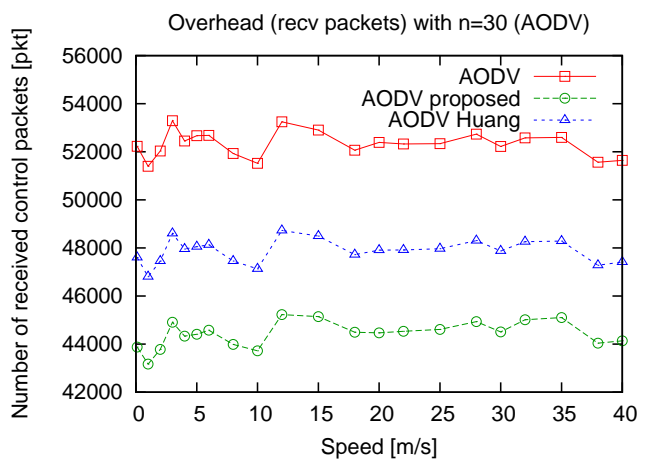

(b) High density $n=30$.

Figure 4: AODV comparison of the received overhead by the network.

It is desirable to maintain small amounts of overhead to reduce energy consumption or congestions in the network. It is equally important to maintain a small value for the CBR drop rate, the better a protocol performs the closer this metric will be to zero.

\subsection{AODV results}

We present in this subsection the results for AODV.

First, from Figure 4 we observe a decrease in the amount of overhead that causes traffic in the network. The biggest decrease is achieved by the approach proposed in this paper. This is due to the fact that our approach reacts to the changes in the network, and when the network is stable (for example at low speeds) fewer Hello messages are sent. Conversely, when the network is not stable, our approach sends more Hello messages to adapt to the changes in the network. There is a concrete reduction of the overhead of almost $30 \%$ compared to the original AODV and almost $15 \%$ compared to Huang's. The three approaches follow a similar tendency due to the fact that 


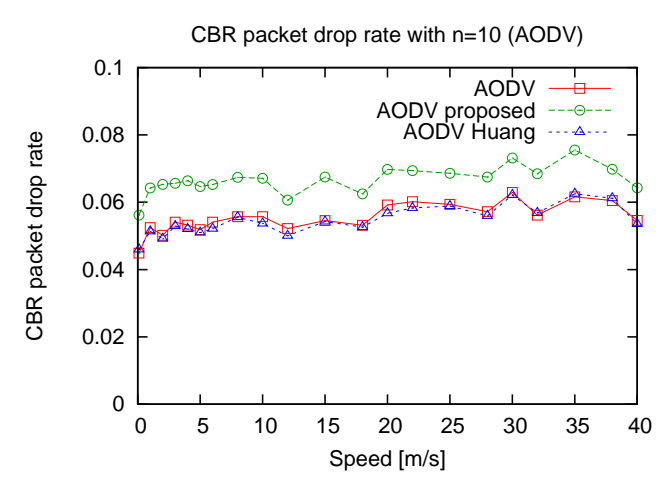

(a) Low density $n=10$.

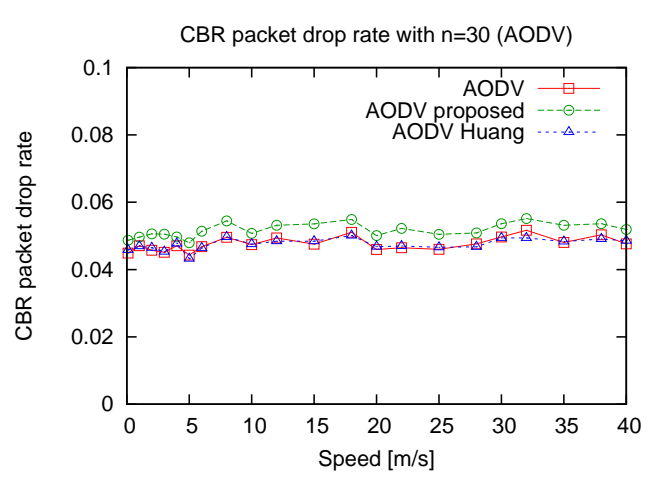

(b) High density $n=30$.

Figure 5: AODV comparison of the CBR drop rate.

the scenarios are the same for each approach. This translates in a similar shape of the curves.

In a low density network, Figure 4 (a), note the tendency of the adaptive schemes (Huang's and the proposed one) to increase the overhead as the speed increases. This is expected because at higher speeds, the network exhibits more changes, and hence the algorithms react sending more Hello messages to detect those changes. This is not the case for high density networks where at high speeds $(40 \mathrm{~m} / \mathrm{s})$, there are many changes in the network and several Hello messages are lost due to collisions. Losing Hello messages degrades the capacity to notice changes in the network. As a result, our approach reacts assuming the network is not changing and does not increase the rate to send Hello messages.

It is equally important to notice that the original AODV has the same amount of overhead regardless of the speed of the nodes, and this is due to the fact that the Hello timer value is always constant.

From Figure 5, we see that there is a slight tradeoff between the overhead 


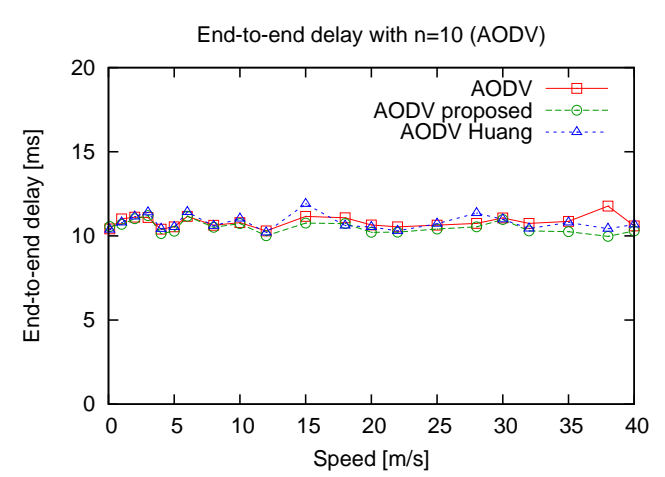

(a) Low density $n=10$.

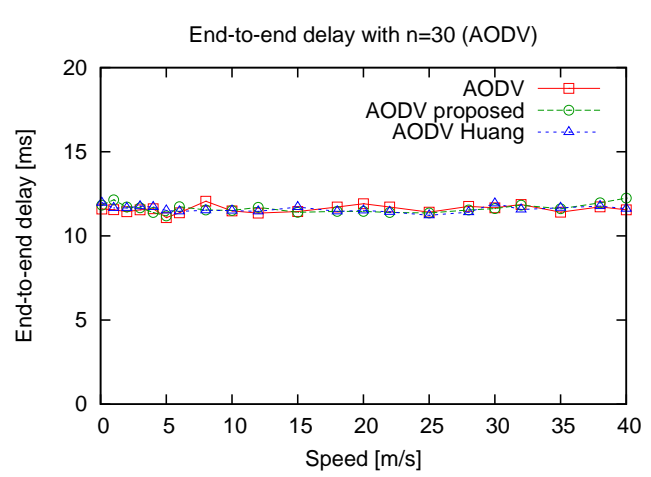

(b) High density $n=30$.

Figure 6: AODV comparison of the E2E delay.

and the CBR drop rate. We observe that the presented scheme is the one that causes the highest CBR drop rate. Compared to the other two approaches, the proposed one has in average less than two percent more CBR dropped packets. From Table 2 we conclude that for low density networks, the CBR drop rate is at most $1.2 \%$ higher in our approach compared to the other two approaches. For high density networks, the CBR drop rate difference between our approach and the other two is less than $0.5 \%$.

From Figure 6, we see that there are small changes in the E2E delay but it is difficult to infer that there is a tradeoff. In fact, we find that there is no significant difference among the three approaches.

In Table 2 we calculate the average values for the performance metrics using 100 simulations and we also calculate $95 \%$ confidence intervals for both populations, $n=10$ and $n=30$ nodes. We observe the advantages of the proposed approach as there is a significant gain in the reduction of the overhead with a small sacrifice in packet loss. Regardless of the approach used, the E2E delay remains practically the same. 
Table 2: AODV performance metrics averages.

\begin{tabular}{|c|c|c|c|c|}
\hline & & Standard & Huang & Proposed \\
\hline \multirow{5}{*}{ Overhead [pkt] } & $\mathrm{n}=10$ & 5494.77 & 4729.41 & 4005.05 \\
\cline { 2 - 5 } & $95 \%$ & 156.86 & 131.58 & 112.85 \\
\cline { 2 - 5 } & $\mathrm{n}=30$ & 52324.08 & 47873.36 & 44425.77 \\
\cline { 2 - 5 } CBR drop rate & $95 \%$ & 806.60 & 717.73 & 6573.19 \\
\hline & $\mathrm{n}=10$ & 0.0554 & 0.0546 & 0.0666 \\
\cline { 2 - 5 } & $95 \%$ & 0.0062 & 0.0062 & 0.0069 \\
\cline { 2 - 5 } & $\mathrm{n}=30$ & 0.0476 & 0.0475 & 0.0517 \\
\cline { 2 - 5 } E2E delay [ms] & $95 \%$ & 0.0035 & 0.0035 & 0.0037 \\
\cline { 2 - 5 } & $\mathrm{n}=10$ & 10.82 & 10.79 & 10.52 \\
\cline { 2 - 5 } & $95 \%$ & 0.79 & 0.73 & 0.63 \\
\cline { 2 - 5 } & $\mathrm{n}=30$ & 11.58 & 11.60 & 11.63 \\
\hline
\end{tabular}

\subsection{OLSR results}

Now we calculate the performance metrics for OLSR. We observe first from Figure 7 that the proposed scheme considerably reduces the amount of overhead that causes traffic in the network. The proposed approach reacts adequately reducing the Hello message sending rate when the network does not change frequently (at low speeds), and reacts increasing the rate when there are more changes (at high speeds). Using our approach, there is a concrete reduction of almost $33 \%$ compared to the original OLSR and almost $11 \%$ compared to Huang's. Note that, for high density networks, higher speeds do not necessarily increase the overhead as there are more collisions in the network. Our approach is, in fact, more sensitive to the packet loss considering that failing to receive Hello messages causes the approach to continue increasing the Hello timer value more aggressively than Huang's approach. As a result, incorrect links lead to higher levels of packet loss. 


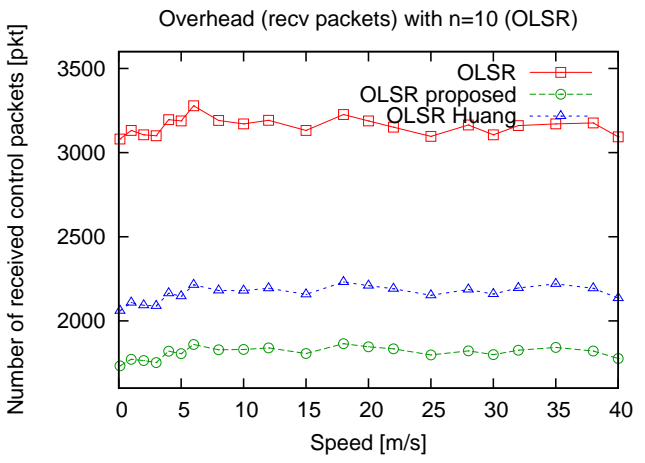

(a) Low density $n=10$.

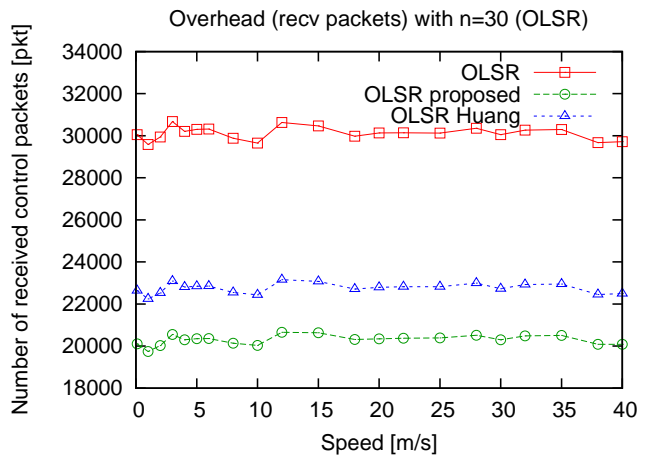

(b) High density $n=30$.

Figure 7: OLSR comparison of the received overhead by the network.

We observe from Figure 8 that there is still a slight tradeoff between the overhead and the CBR drop rate. For low density networks, Fig. 8 (a), Huang's approach and the proposed approach have similar CBR drop rates. However, in high density networks, Fig. 8 (b), the proposed approach exhibits the highest CBR drop rate. In populated networks, the probability to have a collision is higher since there are many more nodes sending packets. This fact raises the CBR drop rate of our approach compared to the other two approaches.

Nevertheless, the differences are very small among the three approaches. In the case of low density networks, the differences are smaller than one percent, and for high density networks the differences are less than $0.5 \%$. Similar to the case for AODV, there is a tradeoff between the CBR drop rate and the overhead reduction. However, using our approach the gain in overhead reduction is more significant than the CBR packet loss rate.

Figure 9 shows that, similar to AODV, the E2E delay in OLSR is not greatly affected by the choice of the scheme. In low density networks, the 


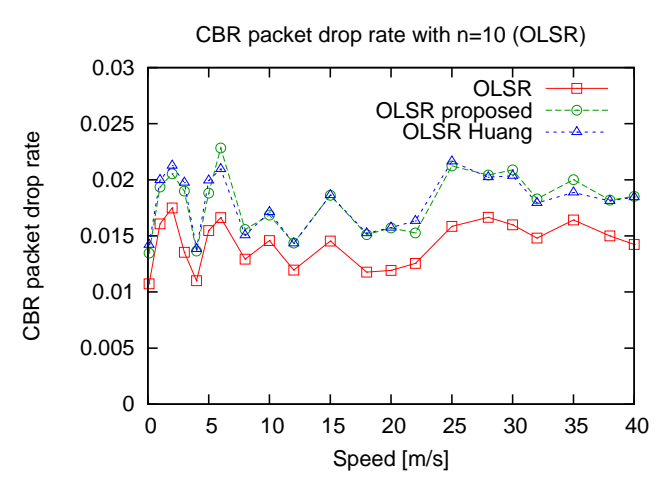

(a) Low density $n=10$.

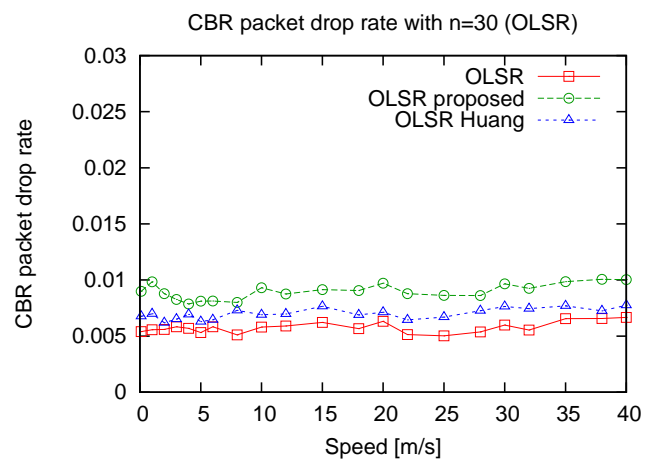

(b) High density $n=30$.

Figure 8: OLSR comparison of the CBR drop rate.

values are similar. However, for high density networks, packet loss causes a larger E2E delay since, contrarily to AODV, an invalid link in OLSR causes more delay due to the recalculation of routing tables. The E2E delay with our approach is in average three milliseconds higher than the original approach and one millisecond higher than Huang's approach.

Finally, similar to AODV, the 100 simulations for OLSR are averaged and 95\% confidence intervals are calculated. The summary is presented in Table 3. 


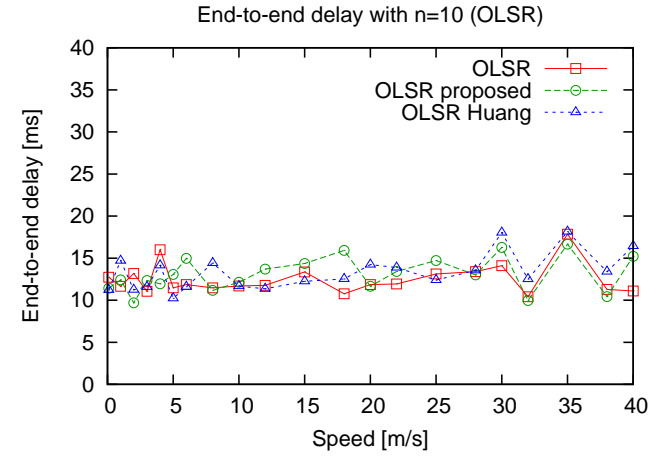

(a) Low density $n=10$.

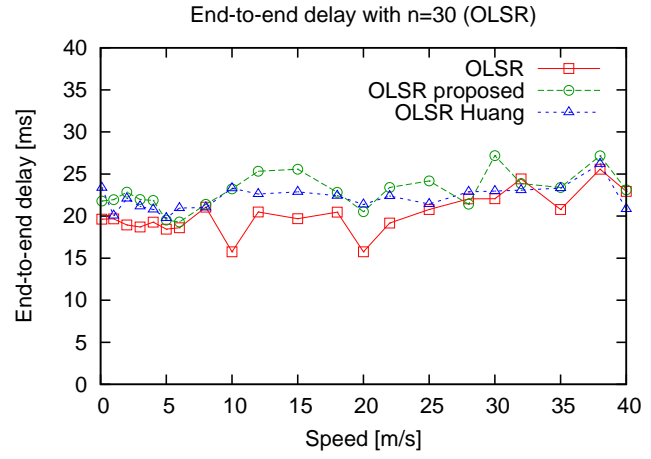

(b) High density $n=30$.

Figure 9: OLSR comparison of the E2E delay.

Table 3: OLSR performance metrics averages.

\begin{tabular}{|c|c|c|c|c|}
\hline & & Standard & Huang & Proposed \\
\hline \multirow{4}{*}{ Overhead [pkt] } & $\mathrm{n}=10$ & 3156.93 & 2164.74 & 1811.20 \\
\cline { 2 - 5 } & $95 \%$ & 90.24 & 58.02 & 47.46 \\
\cline { 2 - 5 } & $\mathrm{n}=30$ & 30117.21 & 22758.31 & 20300.08 \\
\cline { 2 - 5 } CBR drop rate & $95 \%$ & 465.04 & 333.79 & 290.26 \\
\hline & $\mathrm{n}=10$ & 0.0143 & 0.0180 & 0.0179 \\
\cline { 2 - 5 } & $95 \%$ & 0.0041 & 0.0050 & 0.0046 \\
\cline { 2 - 5 } & $\mathrm{n}=30$ & 0.0058 & 0.0070 & 0.0090 \\
\cline { 2 - 5 } & $95 \%$ & 0.0008 & 0.0009 & 0.0012 \\
\hline \multirow{5}{*}{ E2E delay [ms] } & $\mathrm{n}=10$ & 12.48 & 13.31 & 13.07 \\
\cline { 2 - 5 } & $95 \%$ & 2.90 & 3.08 & 3.25 \\
\cline { 2 - 5 } & $\mathrm{n}=30$ & 20.20 & 22.14 & 22.94 \\
\cline { 2 - 5 } & $95 \%$ & 3.48 & 3.90 & 4.07 \\
\hline
\end{tabular}




\section{Conclusions}

In this paper, we considered the problem of the overhead in MANETs. In order to tackle this issue, we proposed a scheme that can help to solve the problem, and validated the effectiveness of the scheme by simulations. The proposed mechanisms can be added as independent modules to the existing protocols, or can be implemented inside the protocols but without changing their architecture or the messages they exchange.

Through simulation experiments using different scenarios, we were able to measure different metrics and compare the overall performance of the proposed approach with the original protocols AODV and OLSR, and another scheme to reduce the overhead proposed by Huang. With the proposed scheme, the reduction of the overhead is greatly achieved with the minimal cost of slightly increasing the drop rate in data traffic. Therefore, the proposed approach outperforms normal standard AODV and OLSR protocols as well as the other approach. The simplicity of our scheme and the independence of any other tools (like GPS) make it suitable for implementations and applications in real scenarios.

For further work, we plan to analyze the dependency between the link change rate and the protocols. We also plan to develop an approach that does not require configuring any parameters.

\section{References}

[1] S. Basagni, I. Chlamtac, V. R. Syrotiuk and B. A. Woodward, "A Distance Routing Effect Algorithm for Mobility (DREAM)," in Proceedings of the 4 th annual ACM/IEEE International Conference on Mobile Com- 
puting and Networking (MOBICOM) '98, Dallas, USA, October 1998, pp. $76-84$.

[2] T. Camp, J. Boleng and L. Wilcox, "Location Information Services in Mobile Ad Hoc Networks," in Proceedings of the IEEE International Conference on Communications (ICC), 2002, pp. 3318-3324.

[3] P. Samar and S. B. Wicker, "On the Behavior of Communication Links of a Node in a Multi-hop Mobile Environment," in Proceedings of the 5th ACM International Symposium on Mobile Ad hoc Networking and Computing (MobiHoc), Tokyo, Japan, 2004, pp. 145-156.

[4] Y. Huang, S. Bhatti and S. Sorensen, "Adaptive MANET Routing for Low Overhead," in International Symposium on a World of Wireless, Mobile and Multimedia Networks (WoWMoM 2007), June 2007, pp. 1-6.

[5] N. Itaya and S. Kasahara, "Dynamic parameter adjustment for available-bandwidth estimation of TCP in wired-wireless networks,", Elsevier Computer Communications, June 2004, vol. 27 issue 10, pp. 976-988.

[6] P. Sharma, D. Estrin, S. Floyd and V. Jacobson, "Scalable Timers for Soft State Protocols," in Proceedings of the Sixteenth Annual Joint Conference of the IEEE Computer and Communications Societies (INFOCOM '97), Kobe, Japan, April 1997, p. 222-229.

[7] C. E. Perkins, E. M. Belding-Royer, and R. Das Samir; Ad hoc OnDemand Distance Vector (AODV) Routing, IETF RFC 3651, July 2003; http://www.ietf.org/rfc/rfc3561.txt. 
[8] I. D. Chakeres and E. M. Belding-Royer, "The utility of Hello Messages for determining Link Connectivity," in Proceedings of the 5th International Symposium on Wireless Personal Multimedia Communications (WPMC), Honolulu, October 2002, vol. 2, pp. 504-508.

[9] T. Clausen and P. Jaquet; Optimized Link State Routing Protocol (OLSR), IETF RFC 3626, October 2003; http://www.ietf.org/rfc/rfc3626.txt.

[10] M. Benzaid, P. Minet and K. Agha, "Integrating Fast Mobility in the OLSR Routing Protocol," in Proceedings of the Fourth IEEE Conference in Mobile and Wireless Communications Networks, Stockholm, Sweden, September 2002, pp. 217-221.

[11] The Network Simulator: ns-2, http://www.isi.edu/nsnam/ns/. 